\title{
A TEST OF FITTS' LAW IN A DUAL-TASK PARADIGM
}

\author{
Randa L. Shehab \\ Robert E. Schlegel \\ University of Oklahoma \\ Norman, OK 73019
}

\begin{abstract}
A simulated automobile driving environment was used to assess the validity of Fitts' Law under dual-task conditions. An aimed hand movement task was used as the Fitts task representative of reaching for controls on an instrument panel. The task required activation of one of four touch-sensitive response plates upon recognition of an auditory stimulus. Movement difficulty was manipulated by varying target location and size. Target location was examined at four levels corresponding to position in a $2 \times 2$ array. Distances of the targets from the twoo'clock position on the steering wheel ranged from $27 \mathrm{~cm}$ to $53 \mathrm{~cm}$. The target plates were square and measured $1.27 \mathrm{~cm}(1 / 2 \mathrm{inch})$ or $0.64 \mathrm{~cm}(1 / 4 \mathrm{inch})$ along the side. The eight combinations of movement amplitude and target size yielded seven unique levels of Fitts' Index of Difficulty (ID) ranging from 5.4 to 7.4. The movement task was performed alone and in combination with two other tasks to create three levels of task loading. A display monitoring task was used to represent the visual demands of driving while an unstable tracking task was used to represent the perceptual-motor demands of driving.
\end{abstract}

Following adequate training, ten subjects performed three replications of six task conditions (three loading levels $x$ two target sizes). Within each replication, the order of testing was counterbalanced across subjects. The dual-task visual loading condition involving the movement and monitoring tasks consistently resulted in the longest reaction times. The dual-task perceptual-motor loading condition involving the movement and tracking tasks resulted in consistently longer movement times. Fitts' ID had a significant effect on both reaction time and movement time for all three conditions of task loading. However, separate linear regressions of movement time on ID for each task loading level resulted in $\mathrm{R}^{2}$ values of 0.66 to 0.82 . Multiple linear regressions involving target size and movement amplitude as predictor variables provided better predictions with $R^{2}$ values of 0.90 to 0.93 . The regression equations provided in this paper may be used by designers to estimate differences in response time due to control size and location.

\section{INTRODUCTION}

In human performance measurement, movement time (MT) has frequently been characterized by a model known as Fitts' Law. Fitts (1954) used information theory to develop an equation to predict movement times for aimed movements. Through a series of three experiments involving reciprocal tapping, disc transfer, and pin transfer tasks, Fitts manipulated movement amplitude (A) and tolerance (target width W). As task characteristics were made more severe, movement time increased accordingly. Fitts developed an Index of Difficulty (ID $=\log _{2}[2 \mathrm{~A} / \mathrm{W}]$ ) which used movement amplitude and tolerance to specify the minimum information required to perform each movement. MT was found to increase linearly with increasing values of ID for serial movement tasks (MT $=a+b$ ID).

Fitts' results were based on repetitive, cyclical tasks where the only degree of uncertainty resulted from the variability in the previous movement's amplitude. Fitts and Peterson (1964) investigated the generality of Fitts' Law for discrete movement tasks. Using a series of five experiments involving discrete movement variations of Fitts' reciprocal tapping task, Fitts and Peterson confirmed Fitts' conclusions. Both movement amplitude and target width were major factors in determining MT, and Fitts' ID was highly correlated with MT. Fitts and Peterson (1964) concluded that ID was equally effective for predicting MT in discrete tasks as well as serial tasks.

Other researchers have confirmed Fitts' Law in different paradigms. Kerr and Langolf (1977) applied Fitts' Law to hand movements in the sagittal plane. Fitts' Law has also been used to describe movement time for the feet (Drury, 1975) and for the head (Jagacinski and Monk, 1985). Chukwu (1990) examined Fitts' Law in a simulated automotive environment under different levels of visual guidance of movement. Chukwu's research confirmed that MT followed Fitts' Law under conditions of direct vision, limited vision, and screened vision (strictly kinesthetic).

One limitation of the previous research is that all studies examined the aimed movement task in isolation. Since single-tasks are rarely encountered in actual humanmachine systems, the applicability of the results is limited. The present study examined the ability of Fitts' Law to describe aimed hand movements performed concurrently with a visual monitoring task or with a perceptual-motor tracking task.

\section{METHODOLOGY}

\section{Experimental Tasks}

The aiming task employed a choice RT method with four stimulus-response alternatives. A speech synthesizer was used to present one of four auditory stimuli to which subjects responded by moving the right hand from the steering wheel and pressing one of four response plates with the right index finger. The response plates were arranged on a $2 \times 2$ array response panel (see Figure 1) mounted on an automobile instrument panel mock-up to the right of the subject. Response plates on a particular panel were all of equal size. Each session of the movement task lasted threeminutes. During this time, stimuli were presented at an average rate of seven per minute. This resulted in a random inter-stimulus interval with a mean of approximately 8 seconds. Using equal numbers of stimuli per position resulted in a minimum of four stimuli per location during a given session.

The task used to induce visual loading was the Display Monitoring (DM) task of the Criterion Task Set (CTS; Shingledecker, 1984). Subjects were required to 


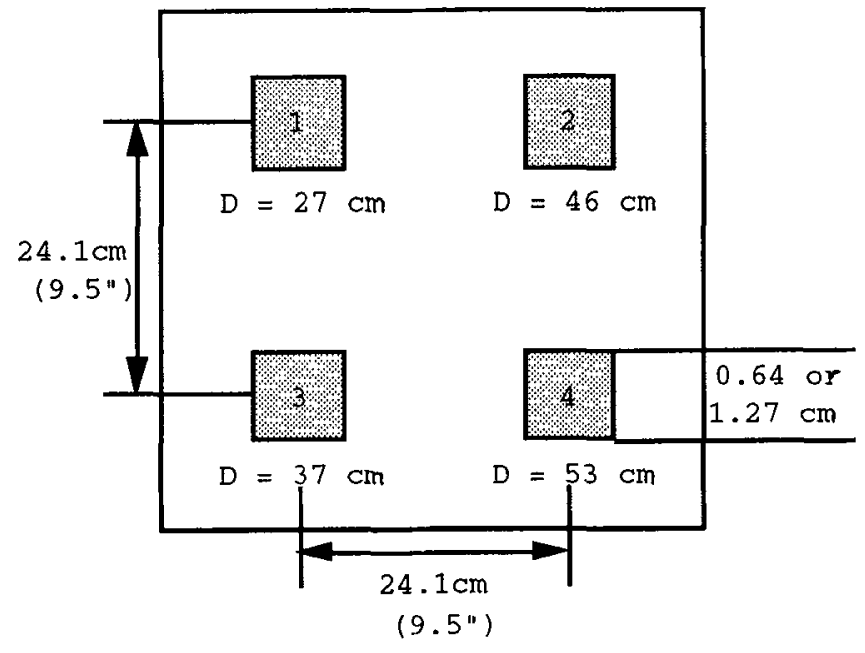

Figure 1. Response Panel $(D=$ distance from two-o'clock position on steering wheel).

monitor two dials on a CRT to determine when a bias signal occurred. Responses were made on a keypad with the left hand. Within a three-minute session, an average of ten signals occurred. The Unstable Tracking (UT) task of the CTS was selected to provide perceptual-motor loading. This task required subjects to stabilize a cursor in the center of a bounded area on the computer monitor using a rotary control with the left hand. The task dynamics are such that control error is magnified, thus creating a condition of instability. The loading tasks were presented on a Commodore 1702 monitor mounted directly in front of the subject at eye level. Both tasks required constant visual attention and/or motor involvement at a level equal to or greater than a moderate driving workload (Schlegel, 1993).

\section{Variables}

Three variables were manipulated in this experiment: task loading, target location, and target size. There were three levels of task loading: (1) the movement task as a single-task, (2) visual loading dual-task combination of the movement task and Display Monitoring, and (3) motor output loading dual-task combination of the movement task and Unstable Tracking. Target plate location was examined using the four locations of the $2 \times 2$ array response panel. Target plate size was either $1.27 \mathrm{~cm}(1 / 2$-inch) square or $0.64 \mathrm{~cm}(1 / 4-i n c h)$ square. Within a session, only one control size was used. The combinations of the target location (i.e., movement amplitude) and target size were also expressed in terms of Fitts' ID as illustrated in Table 1. The primary dependent measures of interest were reaction time (RT) measured from stimulus onset until release of a switch on the steering wheel, and movement time (MT) measured as the time for the movement from the steering wheel to contact with the target.

Table 1. Index of Difficulty as a Function of Movement Amplitude and Target Size.

\begin{tabular}{|c||c|c|}
\hline \multicolumn{2}{|c|}{$\begin{array}{c}\text { Movement } \\
\text { Amplitude (Loc) }\end{array}$} & \multicolumn{2}{c|}{ Target Size (cm) } \\
\hline \hline $26.7 \mathrm{~cm} \mathrm{(1)}$ & 0.64 & 1.27 \\
\hline $46.4 \mathrm{~cm} \mathrm{(2)}$ & 6.4 & 5.4 \\
$35.5 \mathrm{~cm} \mathrm{(3)}$ & 7.2 & 6.2 \\
$53.3 \mathrm{~cm} \mathrm{(4)}$ & 7.8 & 5.8 \\
\hline
\end{tabular}

\section{Experimental Procedure}

Ten subjects with no previous experience on the tasks participated in the three-day study. The first two days were used to train the subjects on all tasks. Training was systematically provided on each task individually and on the dual-task combinations, using all four control locations and both control sizes. On the third day, subjects performed the test sessions. Each subject performed three replications of the six combinations of task loading and control size plus the two CTS tasks individually. Within each replication, the order of testing was counterbalanced across subjects.

\section{RESULTS}

\section{Analysis of Variance}

Training. The first step of the analysis was to examine task learning. Visual inspections of both the RT and MT data from nineteen training sessions indicated that performance had stabilized during training. Analysis of variance on the test data confirmed that session number was not significant for either RT $(F(17,153)=0.56, p=0.92)$ or MT $(F(17,153)=0.72, p=0.78)$. Thus, it was confirmed that performance was stable and session number was excluded from other analyses.

Main Effects. Summaries of the main effects are provided in Figure 2 and Table 2. Repeated measures ANOVA was used to examine the three main factors and all possible interactions. The effect of location was significant $(p<0.0001)$ for both RT and MT, with a Tukey test indicating that location 1 (upper-left) was significantly faster for both measures. For RT, there are two possible explanations. First, the physical mock-up was such that location 1 was more perceptible in the subject's peripheral vision. Second, the shorter distance to location 1 (and thus the lower IDs) required a shorter motor programming time. The explanation for the MT data is also distance related. Targets in the left column of the array had significantly shorter MTs than targets in the right column. In addition, the targets within the left column differed significantly. This finding tends to suggest columnar distance may be as appropriate a measure and provide as much information as individual target locations.

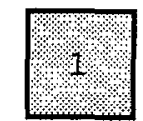

RT $=578$

$\mathrm{MT}=387$

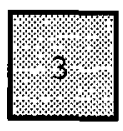

$\mathrm{RT}=651$

$\mathrm{MT}=461$

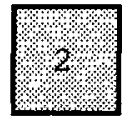

$\mathrm{RT}=654$ $\mathrm{MT}=575$

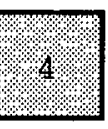

$\mathrm{RT}=625$ $\mathrm{MT}=569$
Figure 2. Reaction Times and Movement Times (msec) as a Function of Target Location. 
Table 2. Mean Reaction Times and Movement Times (msec) by Target Size and Task Load.

\begin{tabular}{|l||c|c||c|c|c|}
\hline \multirow{2}{*}{ Variable } & \multicolumn{2}{|c||}{$\begin{array}{c}\text { Target Size } \\
(\mathbf{c m})\end{array}$} & \multicolumn{3}{c|}{ Cognitive Load* } \\
\cline { 2 - 6 } & 0.64 & 1.27 & AT & AT/DM & AT/UT \\
\hline \hline RT Mean & 624 & 630 & 667 & 628 & 587 \\
RT S.D. & 76 & 75 & 121 & 84 & 77 \\
\hline MT Mean & 459 & 537 & 501 & 544 & 450 \\
MT S.D. & 121 & 168 & 191 & 236 & 147 \\
\hline
\end{tabular}

* AT - Aiming Task; DM - Display Monitoring;

UT - Unstable Tracking; xx/yy - Dual-Task

Target size also had a significant effect on MT ( $p=$ 0.0003 ). Mean MT was shorter for the $1.27 \mathrm{~cm}$ target size. This result follows the direction predicted by Fitts' Law and the intuition that the larger response plate should be easier to contact.

Task loading was significant for both the RT ( $p=$ $0.0005)$ and MT $(p=0.0125)$ measures. For RT, the only significant difference identified with a Tukey test was that the visual loading condition produced a longer RT than did the movement task alone. Neither condition was significantly different from the perceptual-motor loading condition. Thus, of the various task loading conditions, visual loading had the largest impact on RT.

The results of the Tukey test for the MT data indicated that the perceptual-motor loading condition produced a significantly longer mean MT than did the movement task in isolation. Again, neither task was found to be different from the visual loading condition. These data indicated, as expected, that additional motor output loading caused the greatest interference with response movement.

Figures 3 and 4 present reaction time and movement time, respectively, as a function of Fitts' ID and identified by target location and size. Repeated measures analysis of variance using Fitts' ID instead of control location and target size revealed that both $\mathrm{RT}$ and MT were significantly related to ID $(p<0.0001)$. However, the results of the Tukey tests did not present clear patterns or trends for the relationships. This lack of clarity may be attributed to an anomaly that existed in the data at all levels of task loading.

Two combinations of movement amplitude and target size yielded the same ID values (location 1 with $0.64 \mathrm{~cm}$ targets, and location 4 with $1.27 \mathrm{~cm}$ targets). The data obtained for these combinations exhibited unusual patterns. First, a fairly sharp decline was noted in both RT and MT as ID crossed the duplicate ID region. For RT, this was a result of the lack of significance of target size. For MT, this was due to a smaller size effect than one would predict using Fitts' Law. Second, the MTs for location 1 were considerably shorter than for location 4 . For RT, locations 1 and 4 both appeared to be faster than 2 and 3 . This suggests that the strength of the location effect overshadowed the combined impact of movement amplitude and target size as represented by the variable ID. For the MT data, it is unclear what caused the discrepant results. Again there seems to have been some unique attribute of location 1 that contaminated the movement time results. Disregarding the two anomalous data points, MT was reasonably well represented as a linear function of Fitts' ID for both the single-task and dual-task conditions.

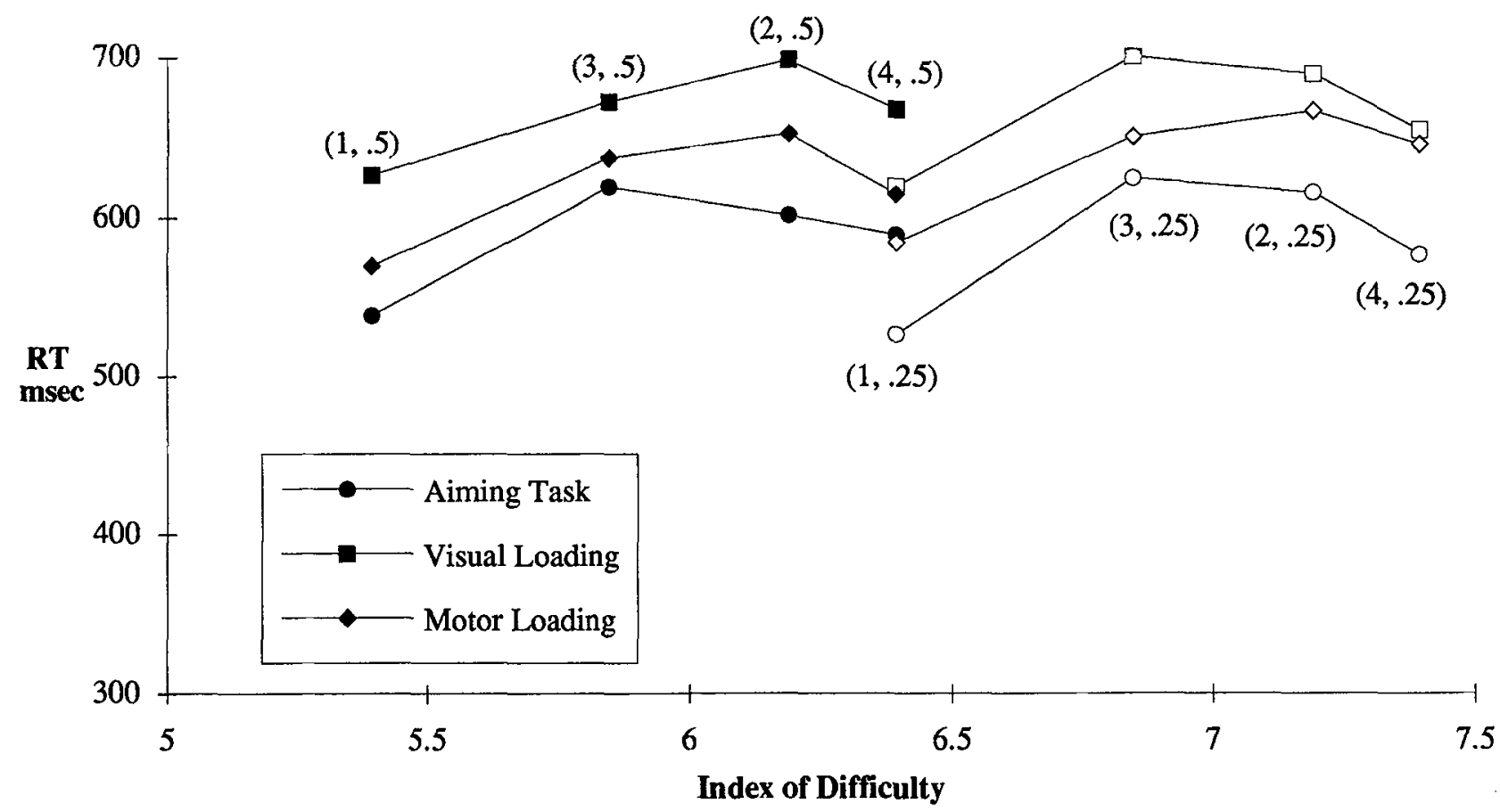

Figure 3. Mean Reaction Time (msec) by ID as a Function of Task Loading. Target Location and Target Size in Inches are Given in Parenthesis by Each Point. 


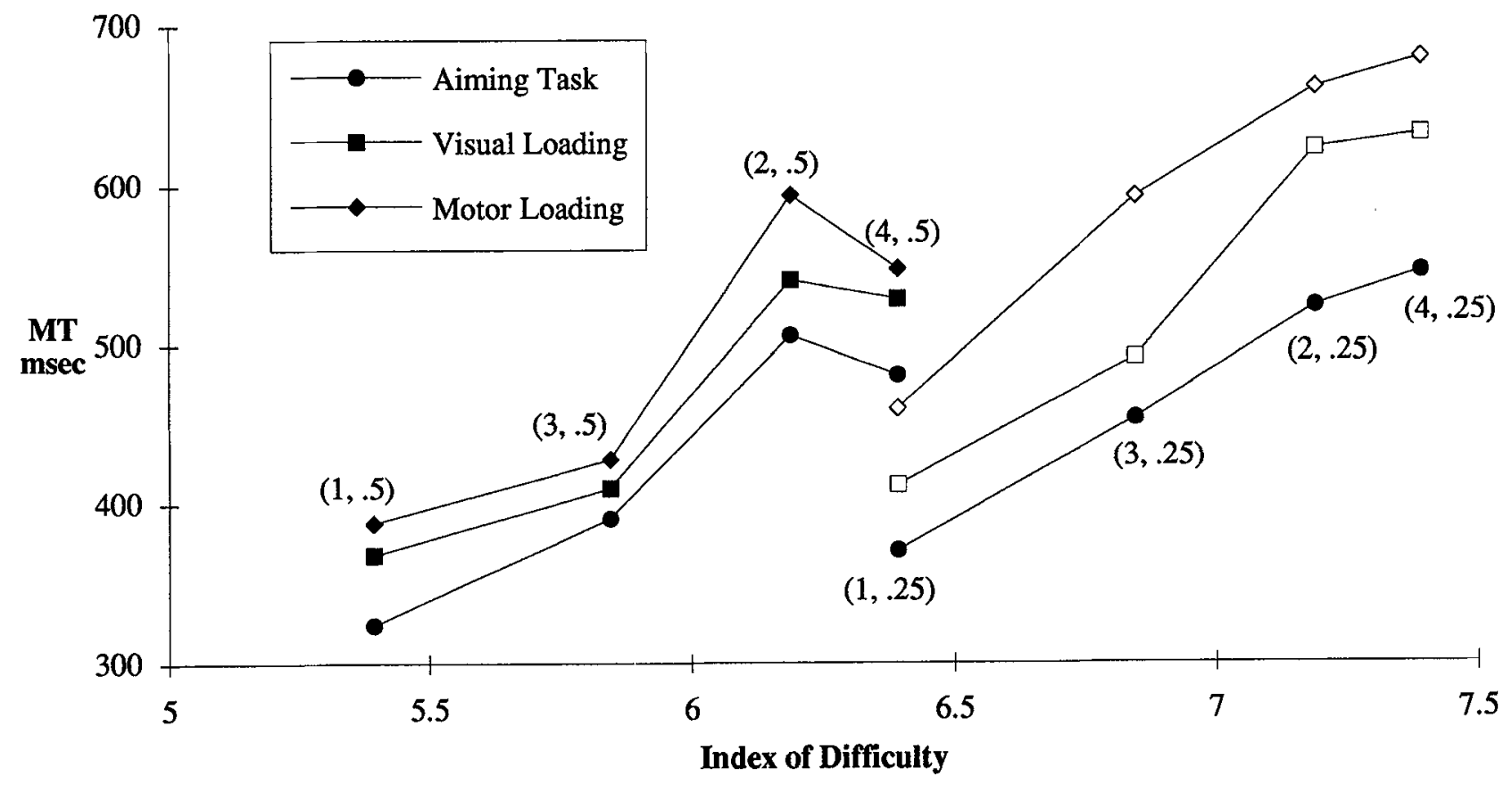

Figure 4. Mean Movement Time (msec) by ID as a Function of Task Loading. Target Location and Target Size in Inches are Given in Parenthesis by Each Point.

\section{Regression Analyses}

Simple linear regressions of RT on ID for each level of cognitive loading were performed. The unclear results of the earlier Tukey test were again reflected in the regression analysis as low values of $R^{2}$. The motor output loading condition yielded the highest value with $\mathrm{R}^{2}=0.43$. However, none of the slope estimates for the regression equation differed significantly from zero. The $\mathrm{R}^{2}$ value obtained for the pure Fitts' task (i.e., the single aiming task) was lower $\left(R^{2}=0.11\right)$, as was the $R^{2}$ obtained for the visual loading condition $\left(\mathrm{R}^{2}=0.13\right)$.

A regression of the means of the MT data vs. ID was performed for each cognitive loading level. The resulting $\mathrm{R}^{2}$ values were considerably higher than those for RT and all ID slope estimates differed significantly from zero. For the single aiming task condition, the model was $M T=-171+96 I D$ with $\mathrm{R}^{2}=0.66(p=0.0142)$. For the visual loading condition, $M T=-315+126 I D$ with $\mathrm{R}^{2}=$ $0.74(p=0.0064)$, and for the motor output loading condition, $M T=-399+146 I D$ with $\mathrm{R}^{2}=0.82(p=$ 0.0019). It is interesting to note that all intercept estimates were negative, and for small values of ID, MT could theoretically be negative. For the values of ID used in this study, this does not pose a problem. In addition, according to Hoffman (1991b), small values of ID represent movements which are ballistically controlled and not appropriately described by Fitts' information theory model. Following Hoffman's line of reasoning, the regression equations would never be used to predict $\mathrm{MT}$ for a movement with such a small ID that MT would be negative.

Multiple linear regression analyses were perforrned for RT and MT as a function of target size and movement amplitude (defined by target location) for each cognitive loading level. The results for RT agreed with those previously found using ID. All of the corresponding $\mathbf{R}^{2}$ values were low and none of the slope estimates reached significance. For the regression of MT, the results using the separate variables were greatly improved over those using ID. The largest impact of the new analysis was evident for the single aiming task. The multiple linear regression model, $\mathrm{MT}=249-193$ Size +17 Distance, now yielded an $\mathrm{R}^{2}$ of 0.93 with the regression coefficients for target size, $(p=0.0427)$ and for movement amplitude $(p=0.0006)$ attaining significance. The model for the visual loading condition was $M T=293-310$ Size +20 Distance with $\mathrm{R}^{2}=0.93(p=0.0181$ for target size and $p=0.0008$ for movement amplitude). For the motor output loading condition, $M T=388-435$ Size +20 Distance with $\mathbf{R}^{2}=0.90(p=0.0134$ for target size and $p=0.0029$ for movement amplitude).

Examination of the equations reveals different trends between the size and distance variables. The coefficient estimates for the size parameter were negative for all levels of cognitive loading. As the task difficulty increased (which for MT indicates a progression from the aiming task to the visual loading task to the perceptual-motor task), the value of the coefficient became increasingly negative. This indicated that the effect of target size became more pronounced as task difficulty increased. This result confirms a significant interaction between target size and cognitive loading identified in the earlier ANOVA $(F(2,18)=4.44, p=0.03)$.

Contrary to the results for target size, the target distance coefficient estimates were all positive, but fairly 
small values. The value obtained for the aiming task was the smallest implying that distance had less of an impact on the aiming task in isolation. In the dual-task conditions, the coefficients were slightly greater, but equal, for both loading conditions. In addition to the implication that distance played a slightly more significant role for the loading conditions than for the single-task condition, this implies that the type of cognitive loading did not impact the effects of target distance. Again, these regression results support the earlier ANOVA which did not find a significant interaction between target location and cognitive loading.

A third set of multiple linear regression analyses was performed which included the interaction of target size and distance in the model. These analyses produced a uniform reduction in $\mathrm{R}^{2}$ values and were, therefore, not considered further.

\section{DISCUSSION}

The original goal of this study was to determine the impact of dual-task loading on the applicability of Fitts Law. Based on ANOVA and regression analyses, it was shown that Fitts' Index of Difficulty could be successfully used to describe aiming performance within a dual-task situation. However, the separate effects of the movement amplitude and target size parameters appeared to provide even better predictions of human movement performance. In addition, a discontinuity was found to exist in the RT and MT data between points of equal ID that varied in movement amplitude and target size. Although the reason for this result is unclear, it may have been due to the minimal effect of target size evident in the regression analysis.

Based on the results of this study, several conclusions can be drawn:

(1) Target location 1 (smallest movement amplitude) had consistently shorter RTs at all cognitive loading levels. This may have been due to the placement of the response panel in the physical mock-up, such that location 1 was more readily visible in the subject's peripheral vision and required less initial motor programming.

(2) Movement amplitudes corresponding to target locations 1 and 3 (the nearest column of the response panel) and the 0.50 inch target size always yielded the quickest MTs.

(3) The visual loading condition elicited the slowest RTs for all movement amplitudes and target sizes. This result was expected on the premise that visual loading directly impacts visual scanning, a component of RT.

(4) The motor output loading condition resulted in the slowest MTs. This confirmed that motor loading caused the greatest interference with response movement.

(5) The interaction of target size and cognitive loading had a significant effect on MT. The increase in MT from the larger to smaller controls was least for the aiming task and greatest for the motor output loaded condition.
(6) ID had a significant effect on RT.

(7) ID had a significant effect on MT.

Although these conclusions were based on a simulated driving task, one could apply this research to the design of control panels in any human-machine system. Prospective panel designs could be evaluated using a dualtask simulation of the system. The impact of the various designs on aimed movement performance as well as system performance could be determined, and thus the optimal control layout or panel design could be selected. Further work in this area should examine a wider range of ID values and investigate the impact of target location in terms of location in the visual field.

\section{REFERENCES}

Chukwu, N.A. (1990). The role of vision and kinesthesis in aimed hand movements. Unpublished Doctoral Dissertation, University of Oklahoma, Norman, OK.

Drury, C.G. (1975). Application of Fitts' Law to foot-pedal design. Human Factors, 17 (4), 368-373.

Fitts, P.M. (1954). The information capacity of the human motor system in controlling the amplitude of movement. Journal of Experimental Psychology, 47 (6), 381-391.

Fitts, P.M., and Peterson, J.R. (1964). Information capacity of discrete motor responses. Journal of Experimental Psychology, 67 (2), 103-112.

Hoffman, E.R. (1991a). Accelerator-to-brake movement times. Ergonomics, 34, 277-287.

Hoffman, E.R. (1991b). A comparison of hand and foot movement times. Ergonomics, 34, 397-406.

Jagacinski, R.J., and Monk, D.L. (1985). Fitts' Law in two dimensions with hand and head movements. Journal of Motor Behavior, 17 (1), 77-95.

Kerr, B.A., and Langolf, G.D. (1977). Speed of aiming hand movements. Quarterly Journal of Experimental Psychology, 29, 475-481.

Schlegel, R.E. (1993). Driver mental workload, in Peacock, B. and Karwowski, W. (Eds.), Automotive Ergonomics (pp. 359-382), London: Taylor \& Francis, .

Shingledecker, C.A. (1984). A task battery for applied human performance assessment research (Tech. Report AFAMRL-TR-84-071). Wright-Patterson Air Force Base, OH: Air Force Aerospace Medical Research Laboratory. 